\title{
Analyse Diagnostique De L'élevage Des Canards De Race Locale (Cairina Moschata Linnaeus, 1758) Dans Le Sud Forestier De La Côte d'Ivoire
}

\author{
Amanidja Boye Donatien \\ Komara Moussa \\ Soro Dofara \\ Loba Akou Logboh Emma Gladys \\ Atcho Otchoumou
}

Laboratoire de Biologie et Cytologie Animales,

UFR des Sciences de la Nature, Université Nangui Abrogoua, Côte d'Ivoire

Doi: 10.19044/esj.2018.v14n36p488 URL:http://dx.doi.org/10.19044/esj.2018.v14n36p488

\begin{abstract}
The duck is one of the indigenous poultry species in Ivory Coast that production is in rudimentary stage. This present study was conducted to identify the systems of breeding, to characterize the number of animal, management practices and the mortality of local ducks through a survey. The survey has been made in participative form of the 375 local duck breeders from the communes of Abobo, Bassam, Bingerville, Port-Bouët and Songon. The survey showed two (02) systems of breeding: an extensive family system with ducks in divagation $(88,62 \%)$ and an extensive family system of farmyard with ducks in captivity with $(11,38 \%)$. The higher breeders $(48,26 \%)$ possesses minus or ten local duck in their flock. Local duck breeders provided a shelter of night (98\%). The factors that decimate the local duck has been domestic accident $(27,75 \%)$, erosion $(34 \%)$ and the predatory $(20,5 \%)$ in the south forest from Ivory Coast. Mortalities has been higher in the females $(30,10 \%)$ than males $(14,98 \%)$. However, duckling $(62,5 \%)$ dead more than the young duck $(20,25 \%)$ or the adults duck $(10,25 \%)$. In conclusion, the local duck breeding is confronted to many constraints, but the installation of rational system of management could help the breeders to improve to increase the productivity of these poultries.
\end{abstract}

Keywords: Breeding, duck, diagnostics analysis, Côte d'Ivoire 


\section{Resume}

Le canard est l'une des espèces indigènes de volaille en Côte d'Ivoire dont la production est encore à l'étape rudimentaire. La présente étude a été menée afin d'identifier les systèmes d'élevage, caractériser l'effectif des animaux, la conduite d'élevage et la mortalité des canards locaux. L'enquête a été faite sous forme participative auprès de 375 éleveurs de canards dans les communes d'Abobo, Bassam, Bingerville, Port-Bouët et Songon. L'enquête a révélé deux (02) variantes du système d'élevage familial extensif: la divagation des canards dans $88,62 \%$ des élevages et la captivité des canards en basse-cour dans 11,38\% des d'élevages. La majorité des éleveurs (48,26 $\%)$ avaient un effectif inférieur ou égal à dix (10) canards. Les éleveurs interrogés fournissaient un abri de nuit (98\%). Indépendamment de l'âge et du sexe, l'érosion (34\%), les accidents domestiques $(27,75 \%)$ et les prédateurs $(20,5 \%)$ sont les principaux facteurs décimant la population de canards dans le Sud forestier de la Côte d'Ivoire. Les mortalités sont plus élevées chez les femelles $(30,10 \%)$ qui est le double de celui des mâles (14,98\%). En outre, les canetons $(62,5 \%)$ meurent plus que les jeunes $(20,25 \%)$ et les adultes $(10,25 \%)$. En conclusion, l'élevage de canards locaux est confronté à de nombreuses contraintes mais la mise en place d'un système de gestion rationnelle pourrait aider les éleveurs à améliorer les performances et accroitre la productivité de ces volailles.

Mots-clés: Elevage, Canard, Analyse diagnostic, Côte d'Ivoire

\section{Introduction}

La Côte d'Ivoire est fortement tributaire du commerce international en matière d'approvisionnement en produits animaux. Ainsi, le pays importe 60 $\%$ des besoins de la population en viande (Miparh, 2009). Dans l'optique de combler le déficit en protéine animale l'Etat ivoirien a orienté sa politique de développement des produits animaux sur la production des animaux à cycle court dont la volaille. Ainsi, des études d'amélioration de la productivité du poulet de race locale dans les élevages traditionnels ont été réalisées (Gnandji,1999; FAO, 2008; Kouadio et al., 2010). Cependant, aucune étude à notre connaissance n'a porté sur l'élevage de la race locale de canards (Cairina moschata). Effet, le rapport de la FAO (2008) cite les canards comme une des espèces aviaires rencontrées en milieu rural. Toutefois, aucune statistique fiable ne permet de determiner l'effectif et les conditions d'élevage du canard en Côte d'Ivoire. De ce fait, bien que l'élevage des volailles de race locale couvre l'étendue du territoire nationale, la place cette espèce reste encore mal connue par les décideurs. Pourtant l'élevage des canards de race locales constitue une importante source de revenu pour les paysans (Fasina et al., 
2007) et représente l'une des rares opportunités d'épargne et d'investissement (Sharmar, 2007). C'est pourquoi notre étude a porté sur l'identification des systèmes d'élevage, la caractérisation de l'effectif, la conduite d'élevage et la mortalité des canards de race locale à travers une enquête dans le Sud forestier de la Côte d'Ivoire.

\section{Matériel et méthodes}

\section{Zone d'étude}

La présente étude a été menée dans le Sud forestier de la Côte d'Ivoire (Figure 1). Cette zone fait partie du domaine Guinéen. Le climat correspondant est de type subéquatorial chaud et humide. Ce type de climat est caractérisé par une grande saison des pluies qui s'étend de Avril à Juillet, puis une petite saison des pluies de Septembre à Novembre. Cette zone connait aussi deux saisons sèches, une grande saison qui débute à partir de Décembre et se termine en Mars. La petite saison sèche couvre le mois d'Août. La température est d'environ $27^{\circ} \mathrm{C}$ et le degré d'hygrométrie annuel moyen est supérieur à 80 $\%$. Les précipitations sont abondantes et peuvent atteindre $1500 \mathrm{~mm}$ d'eau.

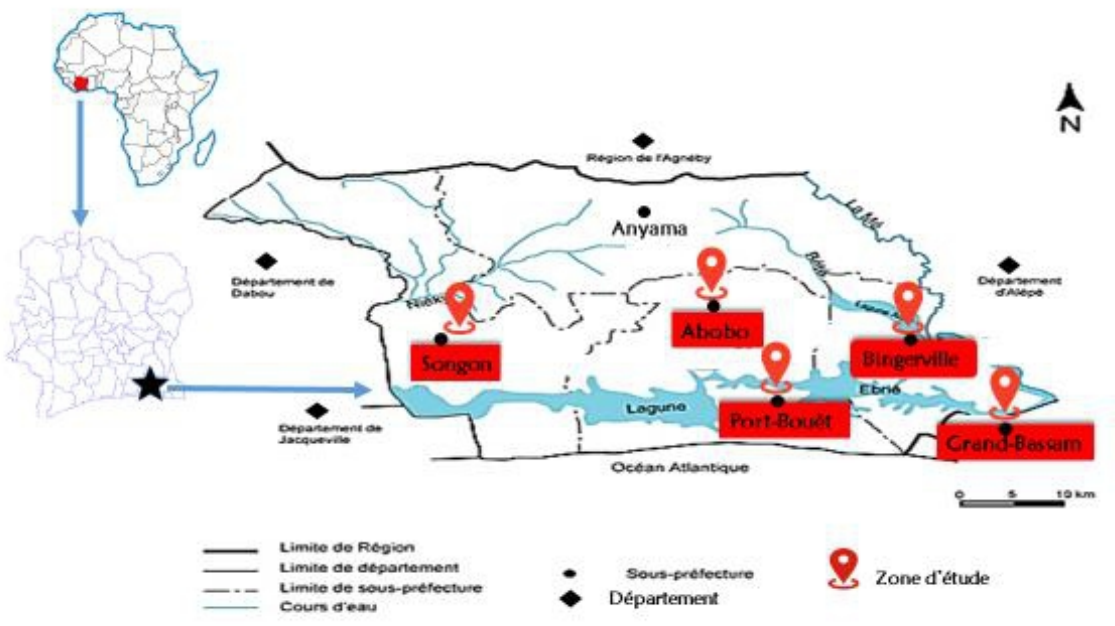

Figure 1: Localisation géographique des zones d'études.

Source: BNETD, 2013 modifié.

\section{Matériel d'étude}

Le matériel d'étude était constitué de fiches de questionnaire d'enquête et d'un appareil photo numérique de marque Samsung pour la prise d'image. 


\section{Méthode}

\section{Elaboration des questionnaires}

Les questionnaires ont été élaborés par rapport aux modes d'élevage des paysans dans le monde rural. L'objectif de ces questionnaires était d'obtenir toutes les informations nécessaires pour la réalisation de cette étude. Le questionnaire a pris en compte les interrogations relatives à l'identification de l'éleveur, à la date de l'enquête, aux caractéristiques de la ferme, à la conduite d'élevage, aux pathologies rencontrées, aux médicaments administrés et à la commercialisation des animaux.

\section{Choix des communes et pré-enquête}

Le choix des communes et des villages a enquêté dans cette étude a été faite selon la méthode employée par Oguntunji olunsegun (2015). Une préenquête a été organisé dans dix (10) ménages dans les villages de chaque commune a visité dans le sud forestier de la Côte d'Ivoire. Au cours des visites, une séance d'échange a porté sur le questionnaire préalablement établi. Cet échange a permis la familiarisation des paysans à cette opération, mais également la modification du questionnaire puis le choix des éleveurs.

\section{Choix des éleveurs}

Le choix des élevages à enquêter, a porté essentiellement sur les éleveurs de canard de la race locale dans le sud forestier de la Côte d'Ivoire. Cependant, aucun critère de sélection n'a été pris en compte en ce qui concerne la taille et l'ancienneté du cheptel de l'éleveur. Par conséquent, nous avons choisi de réaliser l'enquête dans 25 villages de 5 communes du Sud forestier de la Côte d'Ivoire.

\section{Administration des questionnaires}

L'administration des questionnaires s'est déroulé auprès de chaque éleveur sous forme d'enquêtes participatives. Des guides locaux ont été employés pour interpréter et expliquer les questions en langue locale à des paysans qui ne pouvaient pas lire et écrire la langue française. Ainsi, le questionnaire conçu a été soumis aux éleveurs.

\section{Traitement et analyses statistiques des données}

Les analyses statistiques ont été effectués à partir du logiciel $\mathrm{R}$ version 2.12.1. Le test $\mathrm{G}$ qui est l'équivalent du test de Khi deux $(\chi 2)$ a permis de comparer les pourcentages obtenus. Les pourcentages ont été considérées significatives au seuil de 5\%. 


\section{Résultats}

\section{Identification des élevages enquêtés}

Le tableau I montre les communes (05), les villages (25) et le nombre d'éleveurs interrogés (375) au cours de l'enquête.

Taleau I: Elévages des localités enquêtées

\begin{tabular}{|c|c|c|c|c|}
\hline & Communes & Villages & $\begin{array}{l}\text { Nombre } \\
\text { d'éleveurs } \\
\text { interrogés }\end{array}$ & $\begin{array}{l}\text { Nombre } \\
\text { total* }\end{array}$ \\
\hline \multirow{5}{*}{\multicolumn{2}{|c|}{ Abobo }} & Dokui & 06 & \multirow{5}{*}{58} \\
\hline & & Akeikoua & 10 & \\
\hline & & N'dotré & 19 & \\
\hline & & Banco & 10 & \\
\hline & & Allokoi & 13 & \\
\hline \multirow{5}{*}{\multicolumn{2}{|c|}{ Bassam }} & Mondoukou & 08 & \multirow{5}{*}{75} \\
\hline & & Vitré & 14 & \\
\hline & & Nani & 20 & \\
\hline & & Mafiblé & 19 & \\
\hline & & Nanzé & 17 & \\
\hline \multirow{5}{*}{\multicolumn{2}{|c|}{ Bingerville }} & Santé & 12 & \multirow{5}{*}{78} \\
\hline & & Ana & 12 & \\
\hline & & Campement & 19 & \\
\hline & & koroghodougou & 22 & \\
\hline & & Adjamébingerville & 13 & \\
\hline \multirow{5}{*}{\multicolumn{2}{|c|}{ Port bouët }} & Gonzaqville & 21 & \multirow{5}{*}{86} \\
\hline & & Vridi canal & 12 & \\
\hline & & Bonogosso & 20 & \\
\hline & & Derrière waff & 15 & \\
\hline & & Derrière aéroport & 09 & \\
\hline \multirow{5}{*}{\multicolumn{2}{|c|}{ Songon }} & $\mathrm{Km} 17$ & 20 & \multirow{5}{*}{80} \\
\hline & & Adiopodoumé & 22 & \\
\hline & & Bimbresso & 19 & \\
\hline & & M'bratté & 12 & \\
\hline & & Abiaté & 07 & \\
\hline Total & 5 & 25 & 375 & 375 \\
\hline
\end{tabular}

*Nombre total d'éleveurs interrogés par commune.

\section{Systèmes d'élevages des canards de la race locale}

La figure 2 montre les deux systèmes d'élevages des canards de la race locale rencontrés au cours de l'enquête dans le sud forestier de la Côte d'Ivoire. Les deux systèmes d'élevage correspondent au secteur 4 selon la classification de la FAO. Le système familial extensif avec canards en divagation $(88,62 \%)$, où les éleveurs laissent les animaux divaguer pendant la journée à la recherche de nourriture et le système familial extensif de bassecour avec canards en captivité $(11,38 \%)$. En captivité, les canards sont enfermés dans un enclos ou dans un abri de nuit et bénéficie d'une alimentation au cours de la journée. 


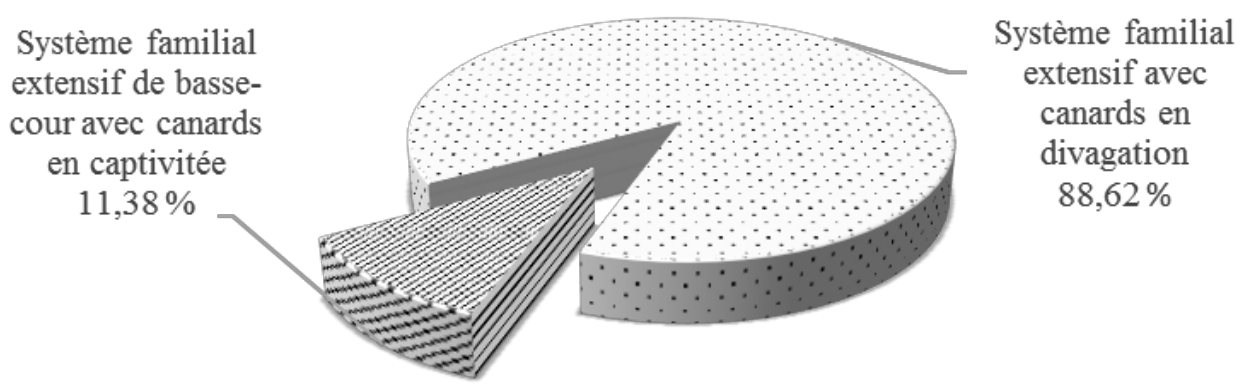

Figure 2: Les deux systèmes d'élevage des canards de race locale rencontrés lors de l'enquête dans le sud forestier de la Côte d'Ivoire.

\section{Effectif et pourcentages des éleveurs de canards de la race locale}

Un effectif total de 5063 canards a été enregistré sur l'ensemble des exploitations. L'effectif total a été subdivisé en sous-effectif en fonction du nombre de canards que disposait chaque éleveur sur sa ferme. En fait, 48,26\% d'éleveurs de la zone d'étude ont un effectif de canards de race locale $\leq 10$. $\mathrm{Au}$ fur et à mesure que l'effectif de canards de race locale augmente la proportion des éleveurs diminue jusqu'à $1,69 \%$ pour des effectifs $>50$. Les effectifs et les pourcentages des éleveurs de canards de la race locale dans les Cinq (05) communes du Sud forestier de la Côte d'Ivoire sont présenté dans le tableau II.

Tableau II: Effectifs et Pourcentages des éleveurs de canards de la race locale dans les Cinq (05) communes du Sud forestier de la Côte d'Ivoire.

\begin{tabular}{lllllll}
\hline \multirow{2}{*}{$\begin{array}{l}\text { Effectif } \\
\text { canards }\end{array}$} & de & \multicolumn{6}{l}{ Pourcentage des éleveurs } & Abobo & Bassam & Bingerville & Songon & $\begin{array}{l}\text { Port } \\
\text { Bouët }\end{array}$ & $\begin{array}{l}\text { Pourcentage total } \\
(\%)^{*}\end{array}$ \\
\hline$\leq 10$ & 52,51 & 38,16 & 43,79 & 54,02 & 52,83 & 48,26 \\
1120 & 41,18 & 27,42 & 28,07 & 15,26 & 23,15 & 27,01 \\
2130 & 5,30 & 18,21 & 15,13 & 17,33 & 14,07 & 14,00 \\
3140 & 2,01 & 12,15 & 7,01 & 6,62 & 4,92 & 6,54 \\
4150 & 0 & 1,27 & 4,98 & 4,52 & 2,1 & 2,50 \\
$>50$ & 0 & 2,79 & 1,02 & 2,25 & 2,93 & 1,69 \\
\hline
\end{tabular}

*Pourcentages total des éleveurs des bandes sur l'ensemble des 5 communes du sud forestier de la Côte d'Ivoire.

\section{Logement et matériel d'élevage des canards de race locale}

Les résultats de l'enquête révèlent que $(98 \%)$ des éleveurs dans les systèmes d'élevage fournissent aux canards un abri de nuit contre $2 \%$ qui n'en fournissent pas. Les abris de nuit sont construits avec différents matériaux 
locaux disponibles dans les villages et d'autres matériaux vendus à moindre coût sur les marchés. Les matériaux utilisés sont les planches, des filets de pêches, des feuilles de tôles ondulées, des bambous fendus, des feuilles de palme, des feuilles de cocotier, des morceaux de briques, du papaux et des bâches (Photographie 1). Le matériel d'élevage utilisé comme abreuvoir sont des bidons en PVC coupés et les abreuvoirs manuels industriels vendus dans le commerce. Les mangeoires sont des bassines, des plateaux en caoutchouc ou en métal et d'autres récipients en forme rectangulaire (Photographie 2).

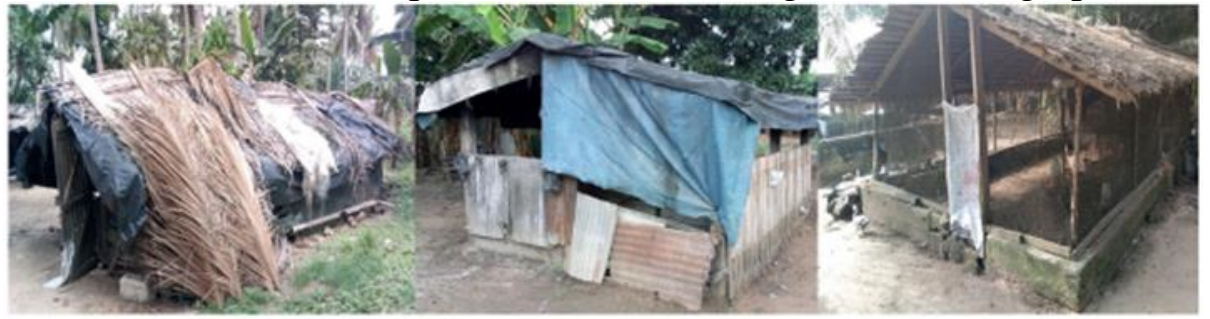

Photographie 1: Différents abris de nuit rencontrés dans les élevages de canards de race locale du Sud forestier de la Côte d'Ivoire.

\section{Alimentation des canards de la race locale}

Les canards élevés dans le système familial extensif en divagation, parcourent les villages à la recherche de nourriture toute la journée. La majorité des éleveurs $(88,62 \%)$ ont leurs canards qui pratiquent cette divagation. Pour les canards élevés dans le système familial extensif en captivité certains éleveurs $(4,28 \%)$ fournissent aux animaux l'aliment industriel de poulet vendu dans le commerce. Tandis que les autres $(7,10 \%)$ nourrissent les canards avec des tubercules de manioc bouillie et du pain mouillé (Photographie 2).

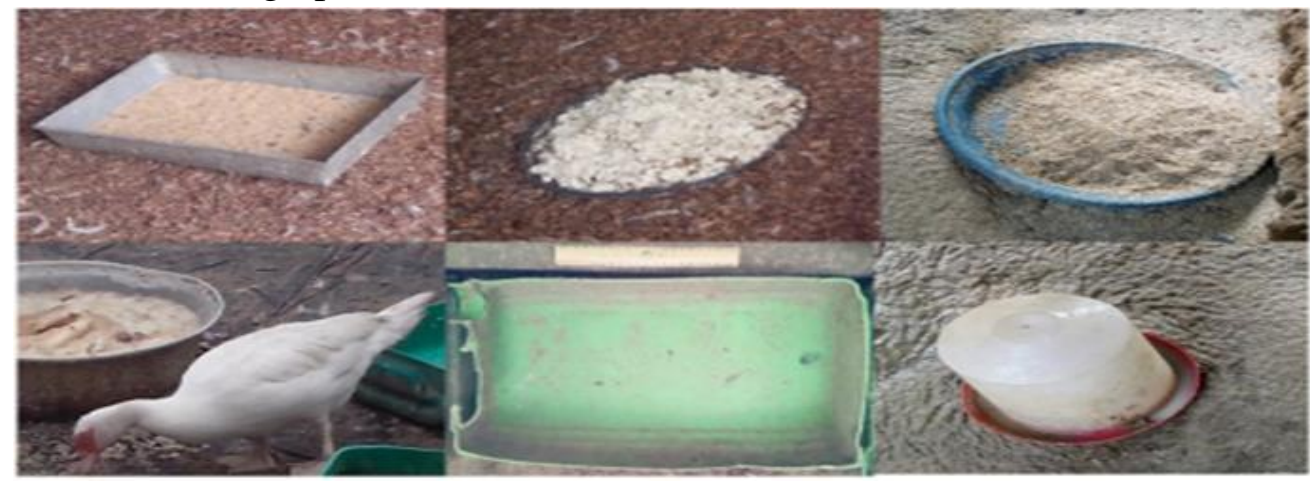

Photographie 2 : Différents aliments et matériels d'élevages rencontrés sur les élevages de canards de race locale du Sud forestier de la Côte d'Ivoire.

\section{Abreuvement et bassin pour canards de la race locale}

Tous les éleveurs interrogés au cours de l'enquête fournissent de l'eau aux canards pour leur abreuvement, mais cette eau provient de sources 
diverses. Ainsi $93 \%$ des éleveurs servent l'eau de robinet contre 7\% d'éleveurs qui servent l'eau de puits. A l'opposée, ce ne sont pas tous les éleveurs qui ont bâtis un bassin pour canards. Le tableau III présente le pourcentage des bassins pour canards rencontrés au cours de l'enquête en fonction des communes.

Tableau III. Pourcentage des bassins rencontrés au cours de l'enquête en fonction des 5 communes du Sud forestier de la Côte d'Ivoire.

\begin{tabular}{ll}
\hline Communes & $\begin{array}{l}\text { Pourcentage }(\%) \\
\text { des bassins }\end{array}$ \\
\hline Abobo & $10,52^{\mathrm{c}}$ \\
Bassam & $16,96^{\mathrm{b}}$ \\
Bingerville & $19,01^{\mathrm{b}}$ \\
Port-Bouët & $28,63^{\mathrm{a}}$ \\
Songon & $24,88^{\mathrm{a}}$ \\
\hline
\end{tabular}

Les valeurs de pourcentages avec des lettres différentes sur une même colonne sont significativement différentes $(\mathrm{P}<0,05)$.

\section{Suivi sanitaire et médicale des canards de la race locale}

En ce qui concerne les règles d'hygiène en dehors du balayage des enclos, aucune désinfection n'est faite lorsque les canards sont en divagation. Les désinfections sont faites uniquement chez les éleveurs ayant les animaux en captivité. Les produits chimiques utilisés par ces derniers sont le Virunet et le Grésil pulvérisés à l'intérieur et aux alentours des bâtiments d'élevage. Sur les 375 exploitations visitées, aucune ferme ne bénéficie d'assistance vétérinaire. Par conséquent, Les éleveurs enquêtés ne disposent d'aucun programme de prophylaxie médicale. Pour le traitement des animaux, les éleveurs pratiquent l'automédication. Ils utilisent soit des anticoccidiens ou des antibiotiques vendus dans le commerce.

\section{Cause des mortalités}

Le pourcentage des causes de mortalités des canards en fonction des facteurs est présenté dans le tableau IV. L'analyse des facteurs responsable de la mortalité indépendamment de l'âge et du sexe a indiqué que l'érosion (34 $\%$ ) et les accidents domestiques $(27,75 \%)$ sont les principaux facteurs décimant les populations de canards dans notre zone d'étude. Des différences hautement significative $(\mathrm{P}<0,05)$ en comparaison avec les autres facteurs ont été observées.

Tableau IV: Pourcentage des causes de mortalités des canards en fonction des facteurs.

\begin{tabular}{ll}
\hline Facteurs & Pourcentages $(\%)$ \\
\hline Accidents domestiques & $27,75^{\mathrm{a}}$ \\
Automobiles & $15,5^{\mathrm{b}}$ \\
chaleur & $0,50^{\mathrm{c}}$ \\
\hline
\end{tabular}




\begin{tabular}{ll}
\hline Maladies & $1,75^{\mathrm{c}}$ \\
Prédateurs & $20,50^{\mathrm{b}}$ \\
Erosion & $34^{\mathrm{a}}$ \\
\hline
\end{tabular}

Les valeurs de pourcentages avec des lettres différentes sur une même colonne sont significativement différentes $(\mathrm{P}<0,05)$.

\section{Sexe et mortalité des canards de la race locale}

Le pourcentage de mortalités des canards en fonction du sexe est présenté dans le tableau V. Le rapport entre le sexe et la mortalité des canards a révélé après analyse que les mortalités étaient plus élevé chez les femelles et par rapport aux mâles. Le pourcentage enregistré au niveau des femelles $(30,10 \%)$ est double de celui des mâles (14,98 \%) avec une différence significative $(\mathrm{P}<0,05)$. Pour 33,5 \% d'éleveurs, les mâles meurent aussi bien que les femelles. A l'opposée, 21,42 \% d'éleveurs n'ont pas pu donner leurs avis concernant le rapport sexe et mortalité.

Tableau V: Pourcentage de mortalités en fonction du sexe des canards de la race locale

\begin{tabular}{ll}
\hline sexe & $\begin{array}{l}\text { Pourcentage }(\%) \\
\text { de mortalités }\end{array}$ \\
\hline Mâle & $14,98^{\mathrm{b}}$ \\
Femelle & $30,10^{\mathrm{a}}$ \\
Mâle et femelles & $33,5^{\mathrm{a}}$ \\
\hline
\end{tabular}

Les valeurs de pourcentages avec des lettres différentes sur une même colonne sont significativement différentes $(\mathrm{P}<0,05)$.

\section{Age et mortalité des canards de la race locale}

Le pourcentage de mortalités en fonction du stade physiologique des canards est illustré par le tableau VI. L'analyse du rapport entre l'âge et la mortalité des canards a indiqué que les mortalités étaient plus élevées chez les canetons que chez les jeunes et les adultes. Les taux enregistrés à chaque stade physiologique de développement ont été respectivement $62,5 \%$ pour les canetons, $20,25 \%$ chez les jeunes et $10,25 \%$ chez les adultes. Cependant, $7 \%$ des éleveurs ne pouvaient pas identifier l'âge précis où les animaux sont le plus prédisposé à la mort. Les taux enregistrés pour le rapport âge et mortalité ont été significativement différent $(\mathrm{P}<0,05)$.

Tableau VI : Pourcentage de mortalités en fonction du stade physiologique des canards de race locale.

\begin{tabular}{ll}
\hline Stade physiologiques & $\begin{array}{l}\text { Pourcentages }(\%) \\
\text { de mortalités }\end{array}$ \\
\hline Canetons & $62,50^{\mathrm{a}}$ \\
Canards jeunes & $20,25^{\mathrm{b}}$ \\
Canards adultes & $10,25^{\mathrm{c}}$ \\
\hline
\end{tabular}


Les valeurs de pourcentages avec des lettres différentes sur une même colonne sont significativement différentes $(\mathrm{P}<0,005)$.

\section{Commercialisation des canards}

Les canards sont vendus à des revendeurs de volailles ou à des particuliers avec des prix fluctuant entre 4000 FCFA et 6000 FCFA pour les femelles, 6000 FCFA et 8000 FCFA pour les mâles. Pour les grands espaces tels que les supermarchés la vente se fait au poids carcasse au prix de 3000 FCFA par kilogramme. Toutefois, les occasions de vente pour les éleveurs sont le plus souvent les périodes de fêtes.

\section{Discussion}

Notre étude montre que les éleveurs de canards pratiquent deux (02) systèmes traditionnels d'élevage : Le système familial extensif avec canards en divagation $(88,62 \%)$, où les éleveurs laissent les animaux divaguer pendant la journée à la recherche de nourriture et le système familial extensif de bassecour avec canards en captivité $(11,38 \%)$. Cette pratique révèle le manque de professionnalisme des éleveurs. Le coût élevé du matériel d'élevage, la mise en place des infrastructures et surtout l'alimentation dans le système intensif serait à l'origine de la pratique du système traditionnel par les éleveurs (Adeyeni et al., 2011). Nos résultats sont similaires avec ceux d'études effectuées chez les canards locaux au Nigéria (Otchere et al., 1990 ; Oguntunji et al., 2015). Ces auteurs ont rapporté au cours de leurs travaux des pourcentages de canards en divagation respectif de $80 \%$ et $86,75 \%$.

Dans notre étude, la quasi- totalité les éleveurs de canards $(48,26 \%)$ ont à majorité des effectifs inférieur ou égal à 10 canards. Ce nombre réduit de la taille des animaux pourrait s'expliqué par le fait que les peuples vivant dans dans le sud forestier de la Côte d'Ivoire sont reconnus pour leur faible attachement à l'élevage au profit des cultures vivrières, industrielles et à la pêche (FAO, 2008). D'où une faible attention accordée par les éleveurs sur ces animaux. Nos résultats sont en accord avec ceux d'études réalisées chez les canards locaux au Nigéria (Duru et al., 2006 ; Nwanta et al., 2006 ; Oguntunji olungesun et al., 2015). Ces auteurs lors de leurs travaux ont rapportés des proportions éleveurs similaire (46,67\%). Cependant, des études conduit au Bengladesh sur des canards de race locale ont enregistré un taux d'éleveurs de 99,3\% ayant des effectifs de canards de race locale compris entre 400 et 1000 chez les paysans éleveurs (Khanum et al., 2005).

Notre étude a montré que les éleveurs sur leur exploitation fournissent aux canards un enclos ou un abri de nuit. Ces derniers sont généralement exigus et ne protègent pas véritablement les animaux contre les prédateurs et les intempéries dû aux conditions climatiques. Quant à l'alimentation, certains éleveurs libèrent, les canards divagués pour se nourrir. Par contre d'autres 
fournissent de l'aliment achetés en commerce $(4,28 \%)$ ou des tubercules de manioc bouillie et du pain mouillé $(7,10 \%)$. Ces conditions d'alimentation pourraient expliquer la faible productivité des canards locaux. Par ailleurs, la distribution d'eau provenait de sources différentes, $93 \%$ des éleveurs utilisent de l'eau de robinet contre $7 \%$ eau de puits protégée. Cette utilisation massive de l'eau de robinet pourrait être mise au compte de l'extension du réseau d'addition en eau potable qui couvre la quasi-totalité du sud forestier de la Côte d'Ivoire.

En revanche, les exploitations ne disposent pas tous de bassin pour canards. La position géographique des villages visités et l'utilisation mixte que font les canards des abreuvoirs mise à leur disposition pourraient être la cause. Pour ces deux raisons, les éleveurs n'accordent aucune importance à bâtir des bassins pour canards. Aussi, la plupart des villages visités sont situés à proximité des branches de la lagune. La lagune sert de lieu de bain aux canards en divagation en présence de forte chaleur. En absence de bassin, les canards se baignent directement dans l'eau d'abreuvement qui leur est servie lorsqu'ils ont chaud. Dans notre étude des pourcentages de bassins allant de 6,89\% à 25 $\%$ ont été enregistré en fonction des communes. Des résultats enquêtes menées par Chang et Villano en (2008) au philippine, Oguntunji et al., en (2015) au Nigéria sur ces mêmes espèces rapportent des pourcentages de bassins différents des nôtres. Ces auteurs ont enregistré respectivement des pourcentages de $76,25 \%$ et de $92,85 \%$ en ce qui concerne la présence des bassins pour canards sur les exploitations.

Dans notre étude, les causes de mortalités des canards indépendamment du sexe a révélé l'érosion comme étant le principal facteur qui décime les effectifs des animaux des éleveurs avec un pourcentage de 34 $\%$. Les fortes précipitations qui s'abattent sur la zone d'enquête pourrait expliquer cette situation. Dans cette zone, les précipitations annuelles peuvent atteindre jusqu'à $1500 \mathrm{~mm}$ de pluie (Sodexam, 2017). Les résultats de ce présent travail étayent la thèse de Nwanta et al., (2006). Cet auteur a notifié selon ces études menées au Congo que c'est l'érosion qui est la cause des mortalités des canards locaux. Par contre, Oguntunji et al., (2015) ont révélé un fort taux de mortalité dû à la maladie $(30,25 \%)$. Le rapport entre le sexe et la mortalité chez les femelles est le double de celui des mâles avec une différence significative. Cela serait dû au dimorphisme très prononcé entre les deux sexes. Cette morphologie permettrait aux mâles de résister aux prédateurs contrairement aux femelles.

Les résultats de notre étude ont révélé que les canetons $(62,50 \%)$ meurent plus que les jeunes $(20,25 \%)$ et les adultes $(10,25 \%)$. La mortalité de ces canetons serait liée aux conditions climatiques du milieu. Après éclosion, les canetons devront faire face aux nombreux défis environnementaux. Il est connu qu'à ce stade de développement les volailles sont dépourvues de glande 
sudoripare, les canetons pouvant mourir suite aux conversion d'énergie effectuée entre le milieu intérieur et le milieu extérieur (froid). Aussi, pendant la divagation ils constituent la proie de certains prédateurs. Les résultats des travaux d'études réalisés au Congo Brazzaville (Banga-m’boko et al.,( 2007) et au Nigéria Chia et Momoh (2012) rapportent ces mêmes observations.

\section{Conclusion}

Il ressort de cette étude que l'élevage des canards de race locale dans le sud forestier ivoirien est réalisé soit par le système familial extensif avec canards en divagation, soit par le système familial extensif de basse-cour avec canards en captivité. La majorité des élevages ont des effectifs de canards inférieur ou égal à 10 animaux. Les mortalités dans ces systèmes sont plus élevées notamment chez les canetons à cause de la mauvaise conduite d'élevage et des conditions climatiques. Au vu de ces résultats, il serait envisageable de former les éleveurs sur les nouvelles techniques moderne d'élevage des canards afin d'améliorer les performances zootechniques et accroître la productivité de ces volailles dans un système d'élevage semiintensif.

\section{References:}

1. Adeniyi, R. \& Oguntunji, O. (2011). Socio-economic survey of cultural practices and management of village poultry production in Ondo area,Nigeria,Livestock Research for Rural Development, 2 (12) : 232-261.

2. Banga-Mboko, H., Maes, D. \& Leroy, L. (2007). Indigenous Muscovy ducks in Congo-Brazaville. A survey of indigenous Muscovy duck management in households in Dolisie city. Tropical Animal Health and Production, 39 (2) : 115-122.

3. Chang, S. \& Villano, R. (2008). Technical and socio-economic constraints to duck production in the philipines: A productivity analysis,International Journal of Poultry sciences, 7 (10) : 940-948.

4. Chia, S. \& Momoh, O. (2012). Some physical and reproductive characteristics of Muscovy ducs (Cä̈rina moschata) under free range mangement system in two locations in Benue state of Nigeria. In : proceedings 37th Animal Conference of Nigerian Society for Animal Production (pp 20-23).

5. Duru, S., Akpa, N. Olugbemi, S. \& Jokthan, E. (2006). A preliminary study on duck mangement under peri-urban system, Livestock Reseach for Rural Development, 18 (3) : 18-36.

6. FAO, (2008). Revue du secteur agricole, Côte d'Ivoire du $1^{\text {er }}$ Décembre 2008, Division de la production et de la santé animales (pp 17-18). 
7. Fasina, F., Wai, D. Mohammed, N. \& Onyekonwu, N. (2007). Contribution de l'aviculture aux revenus des ménages : le cas de la municipalité de Jos South (Nigeria), Aviculture Familiale, 17 (1-2) : 30-34.

8. Gnandji, D. (1999). Troupeaux et cultures des tropiques, Côte d'Ivoire : Amélioration de l'élevage traditionnel de poulet bicyclette (91p).

9. Gueye, F. (2005). Gender aspects of family poultry management systems in developing countries, World's Poultry Science Journal, 61: 39-46.

10. Khanum, H., Chwalibog, A. \& Huque, S. (2005). Study on rural duck production systems in selected areas of Bangladesh, Livestock Research for Rural Development, 17 (10) : 113-117.

11. Kouadio, E., Kouao, J. Fantodji, A. \& Yapi, L. (2010). Influence du système d'élevage sur la performance des poulets locaux de Côte d'Ivoire (Gallus gallus domesticus (Brès, 1973)) de l'éclosion au stade adulte. Journal of applied Biosciences, 32 : 2020-2026.

12. MIPRAH, (2009). Note d'orientation de l'atelier de planification stratégique du plan de relance de l'Aviculture (PASRA) Abidjan, Côte d'Ivoire, Novembre 2009 (8p).

13. Nwanta, A., Umoh, U. Abdu, A. Ajogi, \& L. Ali-Balogun, K. (2006). Management of losses and Newcastle disease in rural poultry in Kaduna State, Nigeria, Journal of Animal Production, 33 (2) : 274285.

14. Oguntunji,O. \& Ayorinde, L. (2015). Duck production : flock characteristics, management and mortality (Caïrina moschata) in Nigeria, Archiva Zootechnica, 18 (1) : 27- 40.

15. Otchere, O., Adeoye, T. Gefu, O. \& Adewuyi, A. (1990). Preliminary observations on village poultry production in North-Central, In : Proceedings of international Workshop on Rural Poultry Development in Africa (Sonaiya, E. B., Ed), Ile-Ife, Nigeria (pp 196-200).

16. Sharmar, K. (2007). Rôle et pertinence de l'aviculture familiale rurale dans les les pays en voie de développement; cas de l'Inde, Aviculture familiale, 17 (1-2) : 35-41.

17. Sodexam, (2017). www. Sodexam.com, consulté le 06/07/2017 